\title{
Carrier power dependence and avoidance methods of passive intermodulation product
}

\author{
Zhao Pei \\ Wireless Theory \& Technology Laboratory \\ Beijing University of Posts \& Telecommunication \\ Beijing, China \\ e-mail:indoor123@sina.com
}

\author{
Liang Tong \\ Research Division \\ China Mobile Group Design Institute Co., Ltd \\ Beijing, China \\ e-mail: liangtong@cmdi.chinamobile.com
}

\begin{abstract}
With the widely development of high power and multi-channel system, passive intermodulaton (PIM) caused by passive components can not be neglected anymore. In this paper, an experimental investigation of the relationship between PIM levels and the carrier powers has been carried out and the results are reported and fitted. Then the performance requirement of passive component used in wireless system is introduced and frequency planning method to avoid effective hit of PGSM is provided.
\end{abstract}

Keywords-passive intermodulation; power variation; avoidance; threshhold;avoidance;

\section{INTRODUCTION}

Passive intermodulation (PIM) means that when two or more signals with different frequency go through the passive component having non-linear characteristics, the $\mathrm{RF}$ signal generated has the relationship with the original signal [1][2].

In order to enhance the system capacity, the using of multiple carriers is widespread in the communication systems, and the power of the carriers also having the trend of increasing. Taking into account that actual circuit usually have non-linear characteristics, intermodulation and intermodulation interference becomes a common phenomenon in cellular mobile communication systems, microwave communication system, a cluster of mobile communication systems, satellite communication systems, ship communication systems, aviation communications systems, cable television systems, etc, this also caused widespread attention.

There are two types of intermodulation, active and passive intermodulation. Given the severity of the intermodulation products, traditionally active intermodulation causes the main concern. But with the application of a more powerful transmitter and the continuous improvement of the receiver sensitivity, the interference caused by PIM growing serious, growing concern is gained by operators, system manufactures and component manufacturers. The characteristics of PIM has been studied in reference [3][4].

In this paper, some basic principles of PIM such as the generation mechanism and affect factors are introduced firstly. Then test the strength of the rule of PIM, and analyze the selection criteria of the passive components. At last, we summarize the intermodulation interference investigation methods.

\section{BASIC PRINCIPLES OF PIM}

Historically, important advances have been made in the development of the general nonlinear models of PIM generation mechanism and the factors that affect PIM [5][6].

\section{A. Generation mechanism of PIM}

When signals with two or more frequency (noted as f1, $\mathrm{f} 2, \ldots, \mathrm{fn}$, called the fundamental) through a nonlinear circuit (the antenna, couplers, etc.) will modulated mutual, these series of mixed products generated by linearly combination of the fundamental wave will appears in the output signal, these mixed product's expression on the frequency is:

$$
k_{1} f_{1}+k_{2} f_{2}+\mathrm{L}+k_{n} f_{n}
$$

where $k_{1}, k_{2}, \ldots, k_{n}$ are integer, these signal is intermodulation product (IMP), $|k|_{1}+\left|k_{2}\right|+\mathrm{L}+\left|k_{n}\right|$ is the order of the intermodulation products. If the intermodulation products just fall within the receiver channel bandwidth, the interference will caused to the receiver, which is the intermodulation interference.

In general, the plus items of each pair of the intermodulation products usually exceed the operating bandwidth; only minus term is likely to fall near the operating bandwidth. Even order intermodulation products, the minus term (such as f0-f1, 2f0-2f1) which are near the DC item will also located outside the operating bandwidth. Therefore, we usually focus on the odd-order intermodulation minus items.

\section{B. Factors that affect PIM}

PIM is caused by the nonlinearity of the component. There are mainly three kinds of modes: first the nonlinearity of the contact, the other is the nonlinearity of the material, finally is the nonlinearity of the process. The nonlinearity of contact means any contact with behavior of the non-linear of current and voltage, such as coaxial cable that is bending uneven, waveguide flange that is not flat, tuning screws and riveting that are loosening, contact with oxidation and corrosion, etc. Material nonlinearity refers to materials with inherent non-linear characteristics, such as ferromagnetic material and carbon fiber. 


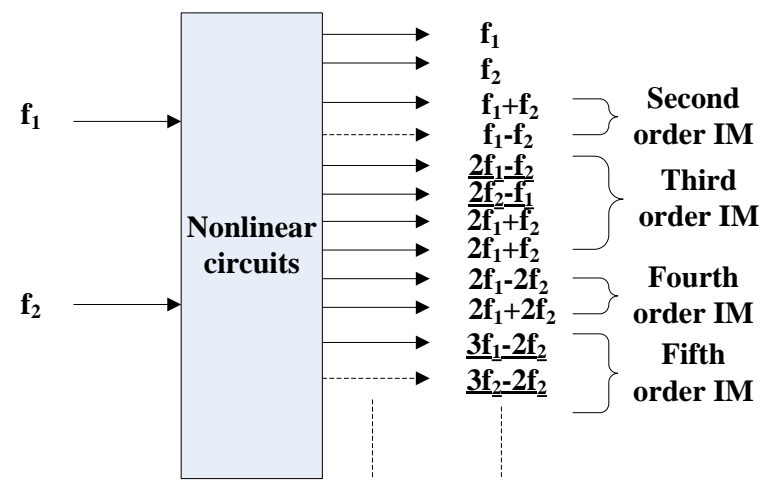

Figure 1. Intermodulation products schematic diagram[6]

Through the analysis of the test procedure and results of PIM interference of antenna systems, it is considered that to mitigate the nonlinearity of the antenna at high power will reduce the intermodulation interference, the nonlinear material should not be used on the antenna and feeder cables [7], such as iron, nickel. The metal to metal connection of the antenna should prevent loose and slip, minimize the use of threaded connection, if condition permits welding is the best. Because the antenna is feed by coaxial cable, the bending degree of the coaxial cable should be as low as possible, so as to avoid large stress at the connection of the cable to avoiding the risks of the generation of the intermodulation interference.

\section{IMPACT OF POWER VARIATION ON PIM}

Reference [8] demonstrates the gradually falling characteristics of the intermodulation products, which means the intensity of higher order intermodulation products is lower than low-order intermodulation. But the strength relationship between the intermodulation products is still the problem in the academic fields, especially for PIM [9][10]. In this section, we select some passive components, to test the 3rd order and 5th order PIM on GSM 900 and DCS 1800, in order to obtain the empirical summary of the strength of PIM.

Based on the capability of intermodulation suppression, these components are divided into two categories, lowPIM (couplers and splitters) and high-PIM (load and attenuator).

The results shows that the 3rd order PIM of low PIM components showing a significant linear relationship with the input power. The test data of the 5th order PIM of the high-PIM components which is meaningful also shows the linear relationship with the input power.

\section{A. Impact of power variation on the $3^{\text {rd }}$ order PIM}

The linear fit coefficients of the 3rd order PIM of these four types of components are in Table I. The fitted curves of the components are in Fig .2 to Fig .5. The accuracy of fitting can be measured by R2. From these figures we can see that,

R2 in most scenes are above 0.91, which means the linear fit of the 3rd order PIM of couplers and splitters are dependent on input power.

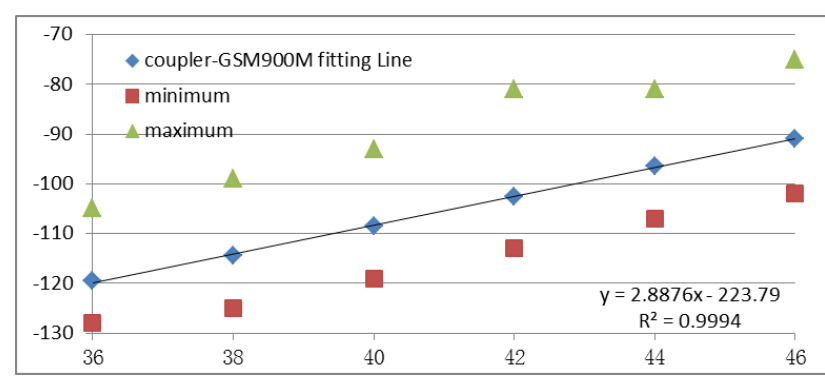

(a)

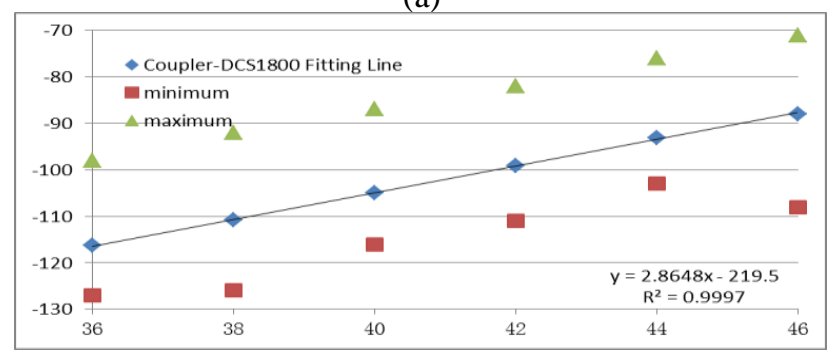

(b)

Figure 2. PIM3 dependent on power/coupler (dBm)

TABLE I. SAMPLES USED IN THE MEASUREMENTS

\begin{tabular}{|c|c|c|c|c|}
\hline \multirow{2}{*}{ Type } & Frequency & $\begin{array}{c}\text { linear } \\
\text { regression } \\
\text { coefficient }\end{array}$ & $\mathbf{R}^{\mathbf{2}}$ & \multirow{2}{*}{ Remark } \\
\hline \multirow{2}{*}{ coupler } & GSM 900 & 2.8876 & 0.9994 & \multirow{2}{*}{5 samples } \\
\cline { 2 - 4 } & GSM1800 & 2.8648 & 0.9997 & \\
\hline \multirow{2}{*}{ splitters } & GSM 900 & 2.6324 & 0.9970 & \multirow{2}{*}{5 samples } \\
\cline { 2 - 4 } & GSM1800 & 2.3371 & 0.9673 & \\
\hline \multirow{2}{*}{ attenuator } & GSM 900 & 2.0857 & 0.9781 & \multirow{2}{*}{ One sample } \\
\cline { 2 - 4 } & GSM1800 & 2.1643 & 0.9990 & \\
\hline \multirow{2}{*}{ load } & GSM 900 & 0.5357 & 0.6142 & \multirow{2}{*}{$\begin{array}{c}\text { 2 samples } \\
\text { of 50W }\end{array}$} \\
\cline { 2 - 4 } & GSM1800 & 0.0664 & 0.7682 & 2 \\
\hline
\end{tabular}

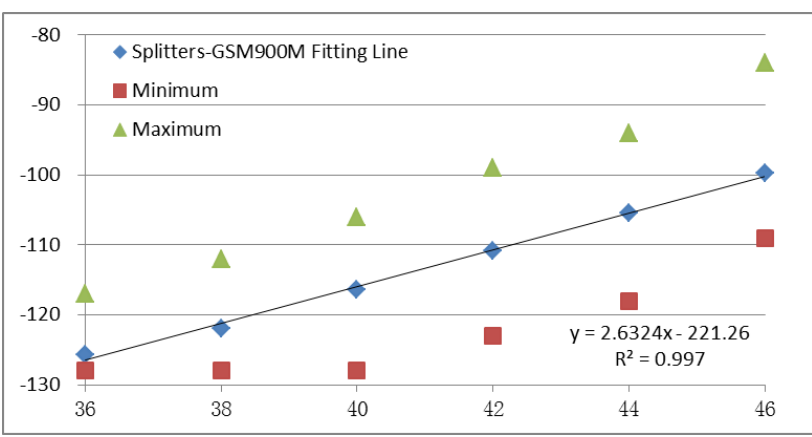

(a)

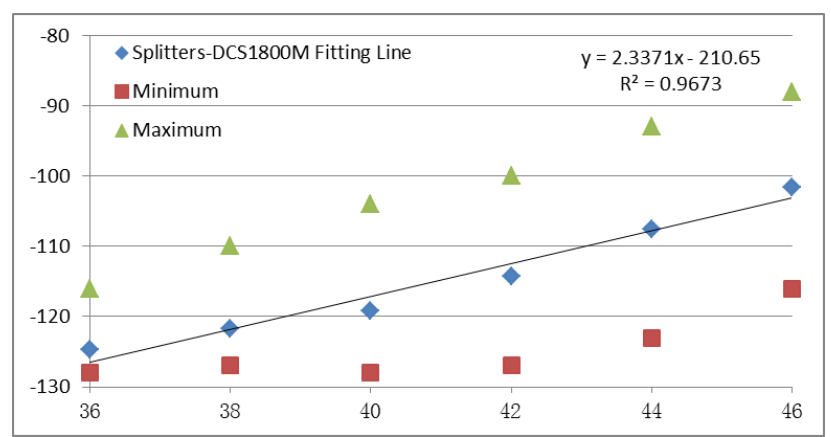

(b)

Figure 3. PIM3 dependent on power/splitter (dBm) 


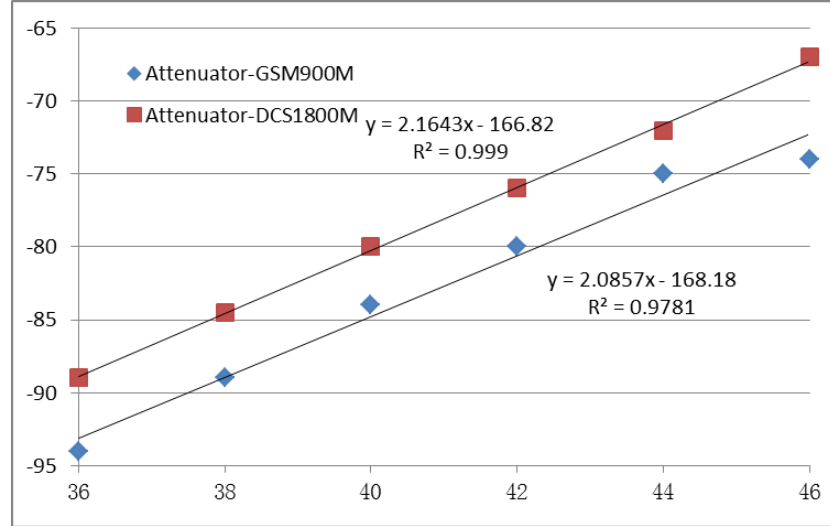

Figure 4. PIM3 dependent on power/attenuator (dBm)

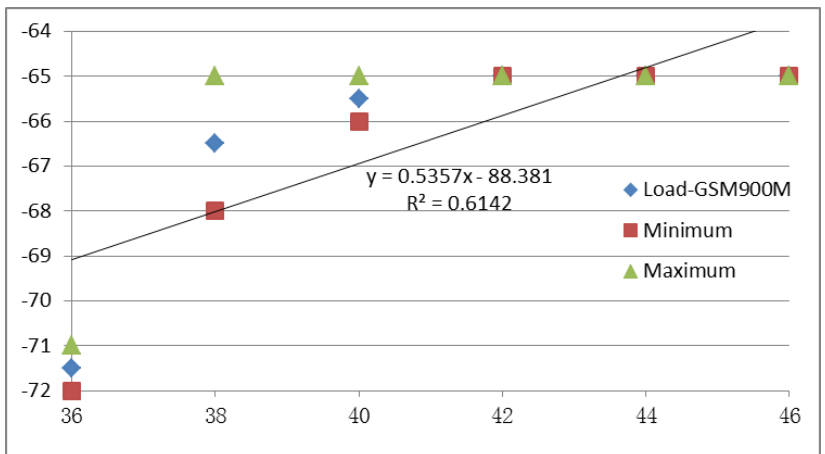

(a)

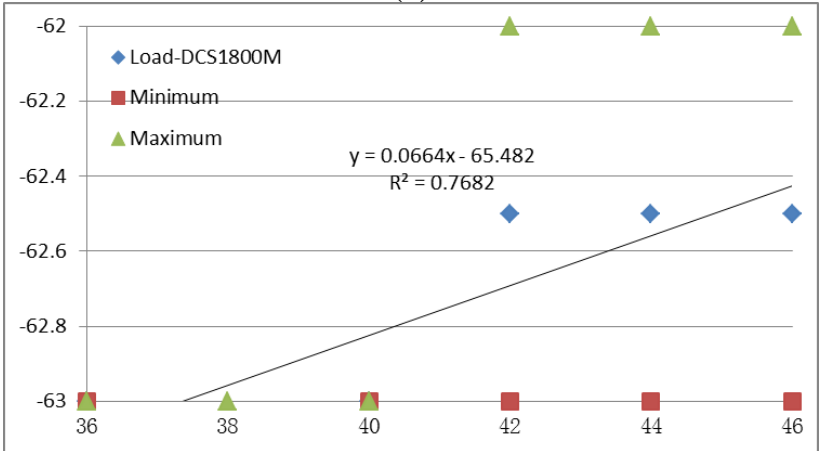

(b)

Figure 5. PIM3dependent on power/load $(\mathrm{dBm})$

- The slope is between 2.1 and 2.9 , that is when input power change $1 \mathrm{dBm}$, the $3^{\text {rd }}$ order of PIM changes about 2.3 2.9 dB. Reference [1] shows the relationship between the $3^{\text {rd }}$ order PIM and the change of the input power, the fitting is more than 0.91 , and the slope is between 0.8 and 2.7.

- By comparing, we can see that the sensitivity of the third order PIM of passive component is more than the coaxial cable.

\section{B. Impact of power vatiation on the $5^{\text {th }}$ order PIM}

The $5^{\text {th }}$ order PIM of the low-PIM components, such as couplers and splitters are all below $-125 \mathrm{dBm}$. But considering that the residual PIM level of the test system we used is only $-168 \mathrm{dBc}$ (which means when the input is $43 \mathrm{dBm}$, the $5^{\text {th }}$ order PIM is $\left.-125 \mathrm{dBm}\right)$. So, the $5^{\text {th }}$ order PIM of the low-PIM components don't have the reference value.

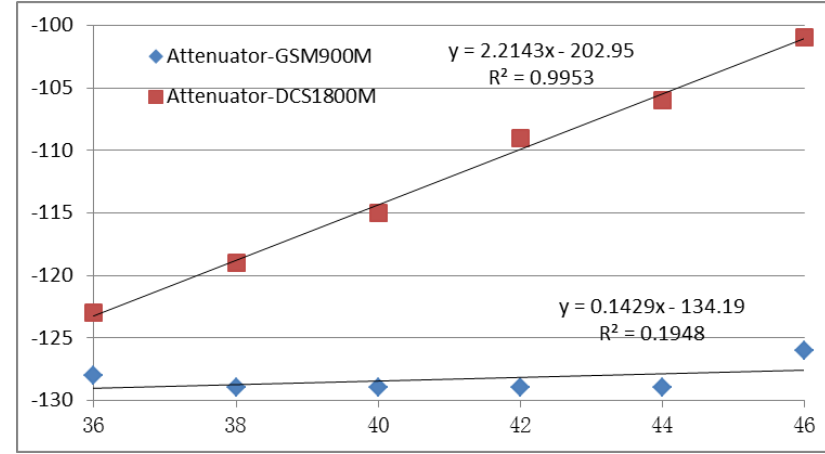

Figure 6. PIM5 dependent on power/attenuator (dBm)

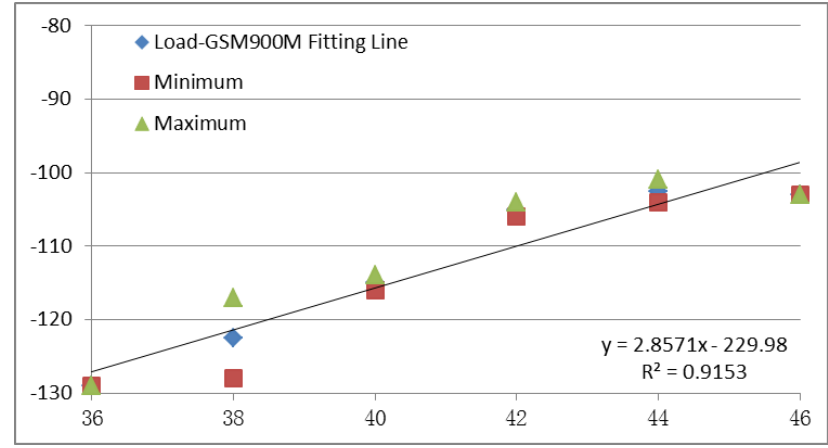

(a)

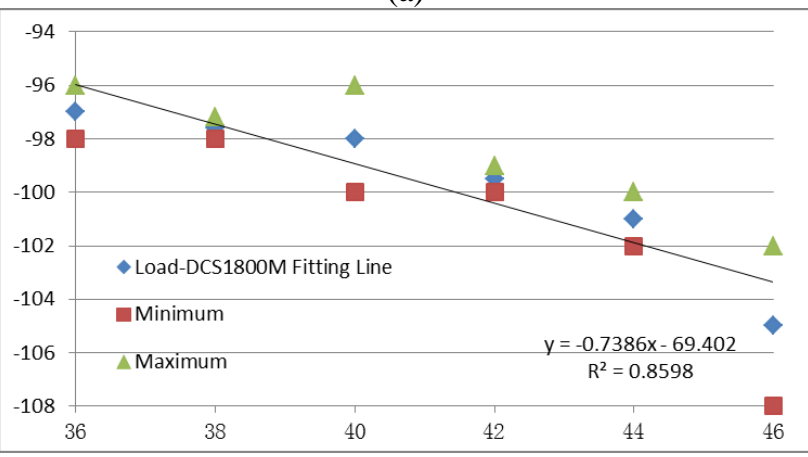

(b)

Figure 7. PIM5 dependent on power/load (dBm)

Fig .6 shows the linear fitting case of the 5th order PIM of the high-PIM components of attenuator. The 5th order PIM of the attenuator on DCS 1800 frequency band shows a trend of obvious more than 2.2 times.

Fig .7 shows the linear fitting case of the 5th order PIM of the high-PIM components of load. The 5th order PIM of the attenuator on GSM 900 frequency band shows a trend of obvious more than 2.9 times. But on the DCS 1800 frequency band shows an anomalous trend. Given that the sample size is small (only two), additional samples need to collect for testing.

\section{Impact of the difference of the $3 r d$ and 5th order intermodulation dependent on power}

The difference between the 3rd order and 5th order PIM of the low-PIM components such as coupler and splitter have the trend of changing with power. When the input is $2 * 43 \mathrm{dBm}$, the difference between the $3 \mathrm{rd}$ order and 5th order PIM for the coupler is $29 \mathrm{~dB}$ and the splitter is $17 \mathrm{~dB}$. It should be noted that, due to the ability of the intermodulation analyzer, the difference below the input 


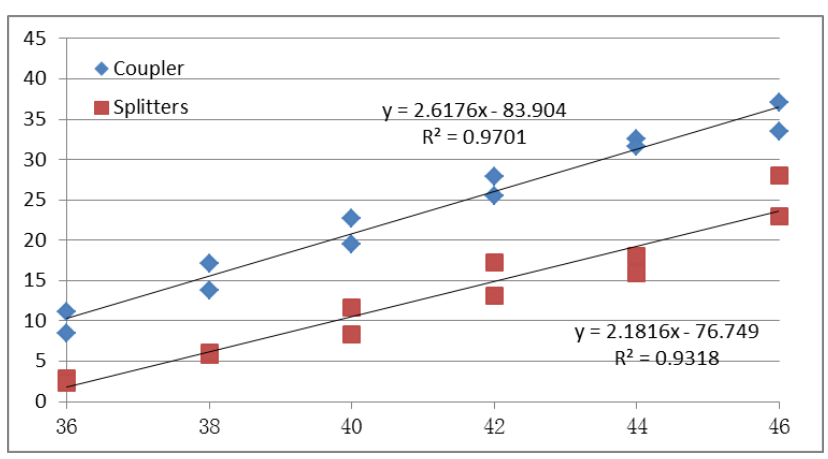

Figure 8. Difference of PIM3 and PIM5 dependent on power $(\mathrm{dBm})$ /coupler and splitter

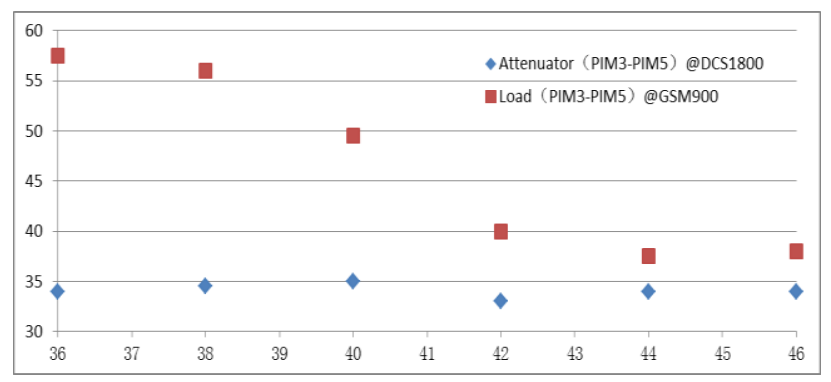

Figure 9. Difference of PIM3 and PIM5 dependent on power $(\mathrm{dBm})$ /attenuator and load

power of $43 \mathrm{dBm}$ is not accurate, and will be small, so the multiple relationships in Fig .8 is to be confirmed.

Fig .9 shows the difference of the 3rd order and 5 th order PIM dependent on the input power. From the figure, when the input power is the same, the difference of the 3rd order and 5th order PIM of the high-PIM components is more than $30 \mathrm{~dB}$.

\section{PIM INTERFERENCE AVOIDING SCHEME}

There are two main means for the circumvention of PIM. The first one is to use high quality component to reduce PIM products radically. The other one is to use the alternatives when the high quality component can't be used for costs or implementation reasons.

\section{A. Principles for design and selection of components}

Conventionally, PIM has been mitigated in systems by choosing low-PIM components. Many studies have been undertaken to identify materials and contact structures conducive to low-PIM operation.

While standards are still under development, an acceptable PIM level is $-150 \mathrm{dBc}$ as the magnitude for $3^{\text {rd }}$ order intermodulation products using two 20-watt transmitters. To achieve this low level of intermodulation, designers are focus on contact design, connector interface, internal junction, cable attachment, materials and plating.

In the following, an explanation of the threshold of -150 $\mathrm{dBc}$ in GSM is provided. The concept of "group intermodulation effect" has been defined in [11], which is used to describe the effect of multi-carriers on the PIM products. And the relationship between the factor of the group intermodulation effect and the carrier number has been defined as follow,

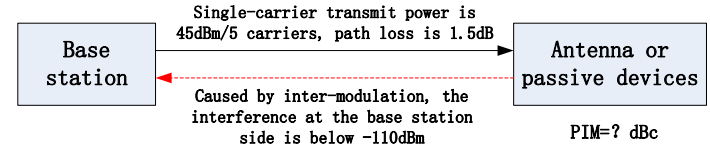

Figure 10. Compute of the intermodulation suppression schematic diagram

$$
\alpha_{n}=10 \times \log [(3 n-7) \times(n-2) / 2]
$$

where, $\alpha_{n}$ is the factor of the group intermodulation effect and $n$ is the carrier number of the system.

Usually, the zero level interference band of GSM is set to $-110 \mathrm{dBm}$. In order to avoid the uplink interference caused by bad quality of components, the PIM interference arriving at the base station through the uplink should not exceed this value. As far as PGSM system is concerned, it has been noted that only the $5^{\text {th }}$ order intermodulation product can result in intra-system interference [2].

The relationship between the single carrier power and the suppression requirements of the $5^{\text {th }}$ order intermodulation can be seen as follow [2],

$$
I=P_{B S}+P_{I M}^{5}+\alpha_{n}-\beta_{5} \times\left(43-P_{i n}\right)-2 L \leq-110
$$

where, $P_{B S}$ is the transmit power of single carrier, $P_{I M}^{5}$ is the suppression capability of the $5^{\text {th }}$ order PIM of the component, $\alpha_{n}$ is the group intermodulation margin, $\beta_{5}$ is the factor of the $5^{\text {th }}$ order PIM dependent on the input power, $P_{i n}$ is the actual input power of the component, $L$ is the path loss.

Since now the average of the carrier numbers of the indoor cell is approximately 8 for most of the metropolitan areas of China, the group intermodulation factor of $\alpha_{n}$ is $17 \mathrm{~dB}$ from Eq. (2). For $\beta_{5}$ is estimated between 2.2 and 2.9 from the test data, 2.5 is used for calculation.

Use the assumption shown in Fig .10, the suppression of the $5^{\text {th }}$ order intermodulation for the first stage of passive components should not above $-170 \mathrm{dBc}$ when the input power is $2 * 43 \mathrm{dBm}$.

The requirement of the antenna is only $-150 \mathrm{dBc}$ for the $3^{\text {rd }}$ order PIM when the input power is $2 * 43 \mathrm{dBm}$, refer to the difference analysis of the $3^{\text {rd }}$ order and $5^{\text {th }}$ order PIM, this requirement for antenna is substantially equivalent to below than $-173 \mathrm{dBc}$ when the power level of the input signals is $2 * 43 \mathrm{dBm}$, which meets the requirement of the above analysis.

The test indicate that, the $5^{\text {th }}$ order PIM of a large number of antennas and passive components is below $140 \mathrm{dBc}$ when the input power is $2 * 43 \mathrm{dBm}$. If the use of frequency is improperly, when single-carrier power increases or multi-carrier combines, great interference will be generated.

\section{B. Frequency planning scheme}

Though many low-PIM components have been developed, the inclusion of low-PIM components into a system is not always desirable or even possible because of other design constraints, such as the large size and the high cost. As an alternative to the conventional methods of 
reducing PIM in wireless system, a kind of frequency planning method can be considered to avoid the intermodulation interference.

In case of only considering the $5^{\text {th }}$ order PIM, through the theoretical calculation, the $19 \mathrm{MHz}$ of GSM 900 band that is authorized to China Mobile, can be divided into three sections, $\mathrm{A}(4 \mathrm{MHz}), \mathrm{B}(8 \mathrm{MHz})$ and $\mathrm{C}(7 \mathrm{MHz})$, as listed in Table II. There will be no effective hit of the $5^{\text {th }}$ order intermodulation if the following principles are followed through careful frequency planning,

(1) Use the frequency in the A, B or $\mathrm{C}$ segment alone.

(2) The frequency in the $\mathrm{B}$ and $\mathrm{C}$ segments can be used in combination.

(3) The frequency in the A and B segments can be used in combination.

(4) Try to avoid using the carriers in the A and C segments simultaneously. Otherwise there's a pretty good chance that effective hit of the $5^{\text {th }}$ order PIM will happen.

TABLE II. ARRANGEMENT TO AVOID EFFECTIVE HIT (PGSM)

\begin{tabular}{|c|c|c|c|}
\hline Category & $\mathbf{A}(\mathbf{4 M})$ & $\mathbf{B}(\mathbf{8 M})$ & $\mathbf{C}(\mathbf{7 M})$ \\
\hline Channel range & $1-20$ & $21-64$ & $65-94$ \\
\hline Frequency range/MHz & $935.2-939$ & $941-947.8$ & $948-953.8$ \\
\hline
\end{tabular}

\section{CONCLUSIONS}

This paper introduces the basic generation principles of PIM, then conduct the strength test of the $3^{\text {rd }}$ order and $5^{\text {th }}$ order PIM on a variety of passive components, the analysis of the strength of the intermodulation is done based on the test data. Finally, in order to avoid PIM, we give two schemes from the requirement quality of the component and the use method of the frequency channels. From the research results, we give the intermodulation requirements for the passive components. In order to avoid PIM, the component meets the requirements should be used. If the component does not meet the requirements, the frequency uses scheme is to be recommended to avoid the intermodulation interference.

\section{ACKNOWLEDGMENT}

We thanks Li Aicheng and Han Chungen did a lot of tests to provide a large number of useful data.

\section{REFERENCES}

[1] Hienonnen S. "Studies on passive intermodulation distortion in antenna structures and design of microstrip antenna elements", Finland :Helsinki University, 2005.

[2] Liang Tong, Zhao Pei, Zhu Yanyun, Meng Dexiang, "The analysis of intermodulation interference for coexistence of different systems including 2G/3G/4G”. General Assembly and Scientific Symposium (URSI GASS),2014 XXXIth URSI , 2014,pp.1-4.

[3] Vicente C, Hartnaged H L. "Passive-intermodulation analysis between rough rectangular waveguide flanges", IEEE transaction on microwave theory and techniques, 2005.

[4] Wilkerson J R, Lam P G, Gard K G, and Steer M B. "Distributed passive intermodulation distortion on transmission lines", IEEE transaction on microwave theory and techniques, 2011

[5] Henrie J, Christianson A, Chappell, W.J. "Prediction of Passive Intermodulation From Coaxial Connectors in Microwave Networks", IEEE Transactions on Microwave Theory and Techniques [J], 2008.

[6] Gao Peng, Zhao Pei, Chen Qingtao, "3G Technical Q \& A, Second Edition," Beijng, People Post Press, June 2011. (in Chinese)

[7] Lijia Chen, Caitian Yang, "The third-order intermodulation interference analysis and measurement of antenna", National antenna symposium, 2009. (in Chinese)

[8] Shiquan Zhang, "Interference studies on microwave and RF frequency passive intermodulation," Xi'an University of Electronic Science and Technology PhD thesis, 2004. (in Chinese)

[9] Hartmut Gohdes, "Impact of power variation on $3^{\text {rd }}$ order passive intermodulation of coaxial RF-cables and their connectors", Proceedings of the 46th International Wire and Cable Symposium, Philadelphia, Pennsylvania, Nov. 17 - 20, 1997.

[10] Justin Henrie, Andrew Christianson, and William J.Chappelll. "Cancellation of passive intermodulation distortion in microwave networks", the 38th European Microwave Conference, 2008,pp.1153-1156.

[11] Cuiyun Tian, "mobile communication system," Posts \& Telecom Press, Beijing, Dec, 1990. (in Chinese) 